Pacific Journal of Mathematic 


\section{ON THE ANALYTIC SPECTRUM OF ARENS}

\section{LUCIEN W AELBROECK}

The object of this note is to show that the "analytic spectrum" of $a=\left(a_{1}, \cdots, a_{n}\right)$ defined by Arens [1] is the complement of the largest open set $X$ in $C^{n}$, on which locally bounded functions, $u_{1}(s), \cdots, u_{n}(s)$ can be found, which satisfy identically the equation

$$
\Sigma\left(\alpha_{i}-s_{i}\right) u_{i}(s)=1 .
$$

We shall have to prove that the existence of bounded functions satisfying this relation in the neighbourhood of the point $t$ implies the existence of analytic functions, which satisfy this equation on a neighbourhood of $t$ that may be smaller than the original one. The main tool in our proof will be statement B, p. 101, of [4].

1. Arens calls a "topological algebra" an algebra, $\mathscr{A}$, with a locally convex structure making the product a continuous mapping of $\mathscr{A} \times \mathscr{A}$ into $\mathscr{A}$, and having further the property that the closed convex span of a compact set is compact. The first thing we shall show is that $\mathscr{A}$ is made into an "algèbre à bornés complète" according to the terminology of [3], when we define as bounded for the structure of $\mathscr{A}$ precisely those sets which are bounded for the topology. It will clearly be sufficient to show that absolutely convergent series converge, that is, that $\Sigma z_{i} b_{i}$ converges when $b_{i}$ is a bounded sequence of $\mathscr{A}$ while $z_{i}$ is a sequence of positive reals such that $\Sigma z_{i}<\infty$.

The sequence of partial sums of the series is obviously a Cauchy sequence. To prove it converges, it is sufficient to show that it is a sequence of elements of a compact set. But we can always find sequences $y_{i}, z_{i}^{\prime}$, which are positive, real, and such that $y_{i} \rightarrow 0, \Sigma z_{i}^{\prime}<\infty$, $z_{i}=y_{i} z_{\imath}^{\prime}$ (when $\left.\Sigma z_{i}<\infty\right)$. We let $b_{i}^{\prime}=y_{i} b_{i}$.

The sequence $b_{i}^{\prime}$ converges to zero. The points $b_{i}^{\prime}$ are the elements of a set whose closure is compact, whose closed convex span is therefore compact. Let $B^{\prime}$ be that closed convex span.

Let $M=\Sigma z_{i}^{\prime}$. The partial sums of the series $\Sigma z_{i} b_{i}=\Sigma z_{i}^{\prime} b_{i}^{\prime}$ belong to $M B^{\prime}$. This proves the series converges. Hence $\mathscr{A}$ is a $b$-algebra.

2. We shall simplify the terminology by calling henceforth the "espaces à bornés complets" and the "algèbres à bornés complètes" of [4]: " $b$-spaces" and " $b$-algebras". The results we shall use being generally true for $b$-algebras, it is interesting to have conditions on an algebra $\mathscr{A}$ on which a locally convex structure is besides defined, which ensure that $\mathscr{A}$ is a $b$-algebra, when we define as bounded the

Received November 28, 1962. 
topologically bounded sets.

In the first place, $\mathscr{A}$ must be a $b$-space. As we have said above, it is sufficient that absolutely convergent series converge, and this condition is not only sufficient, but also necessary.

Further, the product must be bounded. This will of course be the case when the product is a continuous mapping of $\mathscr{A} \times \mathscr{A}$ into $\mathscr{A}$. But weaker continuity conditions on the product are sufficient to ensure its boundedness, when absolutely convergent series converge. In fact, the Banach-Steinhaus theorem shows that seperate continuity is sufficient.

The proof here is exactly the same as that given in [3] in quasicomplete spaces, and in [2] when the algebra is sequentially complete. We can apply Banach-Steinhaus because the hypothesis implies that every bounded set $B$ is included in a bounded set $B^{\prime}$, which is the unit sphere for some complete normed space structure defined on a vector subspace of $\mathscr{A}$.

From now on, $\mathscr{A}$ will be a commutative $b$-algebra with unit. We shall identify the unit of $\mathscr{A}$ with the complex number 1 , and the product of the unit by the number $s$ with $s$ itself.

3. Let $\left(a_{1}, \cdots, a_{n}\right) \in \mathscr{A}^{n}$, let $\left(t_{1}, \cdots, t_{n}\right) \in C^{n}$ (where $C$ is the complex field) and assume that there exist a neighbourhood $\mathscr{U}_{1}$ of $t=$ $\left(t_{1}, \cdots, t_{n}\right)$, and bounded $\mathscr{A}$-valued functions $u_{1}(s), \cdots, u_{n}(s)$ on $\mathscr{U}_{1}$, which satisfy the equation

$$
\Sigma\left(a_{i}-s_{i}\right) u_{i}(s)=1
$$

identically on $\mathscr{U}_{1}$.

We can choose $\varepsilon$ small enough, so that the neighbourhood of $t$ defined by the inequalities $\left|s_{i}-t_{i}\right|<2 \varepsilon(i=1, \cdots, n)$ is included in $\mathscr{U}_{1}$, and shall call $\mathscr{U}_{2}$ the set of $\left(s_{1}, \cdots, s_{n}\right)$ satisfying the inequalities $\left|s_{i}-t_{i}\right|<\varepsilon(i=1, \cdots, n)$.

Two auxiliary $b$-algebras will be useful in the proof. $\mathscr{A}_{1}$ will be the algebra of all bounded $\mathscr{A}$-valued holomorphic functions on $\mathscr{U}_{2}$, a subset $B$ of $\mathscr{A}_{1}$ being bounded when the set $B^{\prime}$ of all $f(z), f \in B$, $z \in U_{2}$ is a bounded subset of $\mathscr{A}$.

We shall also have to consider the algebra called $\Theta\left(s ; \delta_{0} ; \mathscr{A}_{1}\right)$ in [4]. This is the algebra of all $\mathscr{A}_{1}$-valued functions, $\varphi$, of the variables $s$, which are defined on $C^{n}$, and have the property that $\delta_{0}(s)^{N} \varphi(s)$ is a bounded $\mathscr{A}_{1}$-valued functions, for $N$ large enough, when $\delta_{0}(s)=$ $\left(1+|s|^{2}\right)^{-1 / 2}$.

$\mathscr{A}_{1}$ being an algebra of functions of $z, \Theta\left(s ; \delta_{0} ; \mathscr{A}_{1}\right)$ is an algebra of $\mathscr{A}$-valued functions of $(z, s)$ which are defined on $\mathscr{U}_{2} \times C^{n}$, and are holomorphic on $\mathscr{U}_{2}$ for $s$ constant. These functions have further the 
property that $\left(1+|s|^{2}\right)^{-N / 2} \varphi(z, s)$ is a bounded $\mathscr{A}$-valued function when $N$ is large enough. (They have polynomial growth in $s$, uniformly in function of $z$ ).

4. We wish to show that the ideal generated by $a_{1}-z_{1}, \cdots, a_{n}-z_{n}$ in $\mathscr{A}_{1}$ is the unit ideal (i.e. that this ideal contains the unit of $\mathscr{A}_{1}$, and is therefore equal to $\mathscr{A}$ itself). Statement B, p 101, of [4] shows that it is sufficient to prove that the ideal generated by $a_{1}-z_{1}, \cdots$, $a_{n}-z_{n}, z_{1}-s_{1}, \cdots, z_{n}-s_{n}$ in $\Theta\left(s ; \delta_{0} ; \mathscr{A}_{1}\right)$ is the unit ideal.

Bounded $\mathscr{A}$-valued functions $u_{i}(s)$ are given on $\mathscr{U}_{1}$, which satisfy the equation

$$
\Sigma\left(a_{i}-s_{i}\right) u_{i}(s)=1
$$

identically on $\mathscr{U}_{1}$. When $s \in v_{1}$, we write $U_{i}(z, s)=V_{i}(z, s)=u_{i}(s)$.

When $s \notin \mathscr{U}_{1}$, we select an index $j$, depending on $s$, and such that $\left|s_{j}-t_{j}\right|>2 \varepsilon$; we define $U_{i}(z, s), V_{i}(z, s)$ by

$$
\begin{aligned}
& U_{i}(z, s)=0 \text { for all } i \\
& V_{i}(z, s)=0 \text { for } i \neq j \\
& V_{j}(z, s)=\frac{1}{z_{j}-s_{j}} .
\end{aligned}
$$

This defines $U_{i}(z, s), V_{i}(z, s)$ for all $(z, s) \in \mathscr{U}_{2} \times C^{n}$. These are holomorphic functions of $z$, and bounded $\mathscr{A}$-valued functions of $(z, s)$, they belong therefore to $\Theta\left(s ; \delta_{0} ; \mathscr{A}_{1}\right)$. They satisfy identically the equation

$$
1=\Sigma\left(a_{i}-z_{i}\right) U_{i}(z, s)+\Sigma\left(z_{i}-s_{i}\right) V_{i}(z, s) .
$$

And we know that this can only happen when there exist elements $u_{1}^{\prime}(s), \cdots, u_{n}^{\prime}(s)$, in $\mathscr{A}_{1}$, which satisfy the equation

$$
1=\Sigma\left(a_{i}-z_{i}\right) u_{i}(z) .
$$

This is the required result, since the functions $u_{i}$ are holomorphic.

\section{BIBLIOGRAPHY}

1. R. Arens, The analytic functional calculus in commutative topological algebras, Pacific

J. Math., 11 (1961), 405-429.

2. G. Neubauer, Zur spektraltheorie in lokallonvexen algebren, Math. Ann., $142(1960 / 61)$, 131-164.

3. L. Waelbroeck, Note sur les algèbres du calcul symbolique, Journal de Math P. et App., 37 (1958), 41-44.

4. ——, Etude spectrale des algèbres complètes Mémoires Acad. Belgique, Cl. des Sci., 1960. 



\section{PACIFIC JOURNAL OF MATHEMATICS}

\section{EDITORS}

\section{RalPh S. Phillips}

Stanford University

Stanford, California

M. G. Arsove

University of Washington

Seattle 5, Washington
J. Dugundji

University of Southern California Los Angeles 7, California

Lowell J. Paige

University of California

Los Angeles 24, California

\section{ASSOCIATE EDITORS}
E. F. BECKENBACH
D. DERRY
H. L. ROYDEN
E. G. STRAUS
T. M. CHERRY
M. OHTSUKA
E. SPANIER
F. WOLF

\section{SUPPORTING INSTITUTIONS}

\author{
UNIVERSITY OF BRITISH COLUMBIA \\ CALIFORNIA INSTITUTE OF TECHNOLOGY \\ UNIVERSITY OF CALIFORNIA \\ MONTANA STATE UNIVERSITY \\ UNIVERSITY OF NEVADA \\ NEW MEXICO STATE UNIVERSITY \\ OREGON STATE UNIVERSITY \\ UNIVERSITY OF OREGON \\ OSAKA UNIVERSITY \\ UNIVERSITY OF SOUTHERN CALIFORNIA
}

STANFORD UNIVERSITY

UNIVERSITY OF TOKYO

UNIVERSITY OF UTAH

WASHINGTON STATE UNIVERSITY

UNIVERSITY OF WASHINGTON

AMERICAN MATHEMATICAL SOCIETY

CALIFORNIA RESEARCH CORPORATION SPACE TECHNOLOGY LABORATORIES

NAVAL ORDNANCE TEST STATION

Printed in Japan by International Academic Printing Co., Ltd., Tokyo Japan 


\section{Pacific Journal of Mathematics}

\section{Vol. 13, No. 1 \\ March, 1963}

Frantz Woodrow Ashley, Jr., A cone of super-(L) functions............. 1

Earl Robert Berkson, Some metrics on the subspaces of a Banach space....

Felix Earl Browder and Walter Strauss, Scattering for non-linear wave

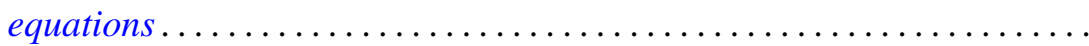

Edmond Darrell Cashwell and C. J. Everett, Formal power series ..........

Frank Sydney Cater, Continuous linear functionals on certain topological

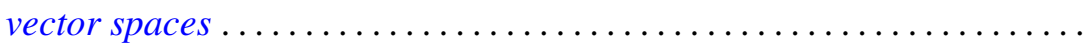

John Douglas Dixon, General group extensions ....................

Robert Pertsch Gilbert, On harmonic functions of four variables with

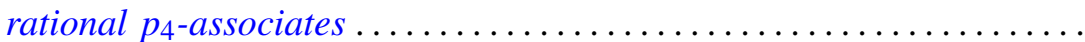

Irving Leonard Glicksberg, On convex hulls of translates ..............

Simon Hellerstein, On a class of meromorphic functions with deficient zeros

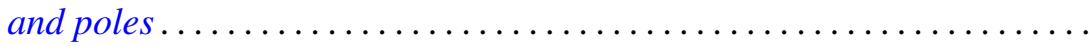

Donald William Kahn, Secondary cohomology operations which extend the

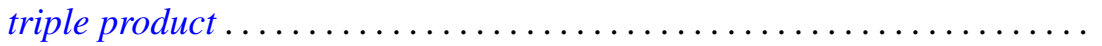

G. K. Leaf, A spectral theory for a class of linear operators .............

R. Sherman Lehman, Algebraic properties of the composition of solutions of partial differential equations ........................... 157

Joseph Lehner, On the generation of discontinuous groups ............. 169

S. P. Lloyd, On certain projections in spaces of continuous functions ...... 171 Fumi-Yuki Maeda, Generalized spectral operators on locally convex spaces ..................................

Donald Vern Meyer, $E^{3}$ modulo a 3-cell

William H. Mills, An application of linear programming to permutation groups.

Richard Scott Pierce, Centers of purity in abelian groups

Christian Pommerenke, On meromorphic starlike functions ...

Zalman Rubinstein, Analytic methods in the study of zeros of

polynomials...

B. N. Sahney, On the Nörlund summability of Fourier series

Tôru Saitô, Regular elements in an ordered semigroup . .

Lee Meyers Sonneborn, Level sets on spheres...........

Charles Andrew Swanson, Asymptotic estimates for limit point

problems .

Lucien Waelbroeck, On the analytic spectrum of Arens . .

Alvin (Murray) White, Singularities of a harmonic function of three

variables given by its series development .............

Kōichi Yamamoto, Decomposition fields of difference sets ...

Chung-Tao Yang, On the action of $\mathrm{SO}(3)$ on a cohomology manifold... 